\title{
ENFERMAGEM NAS URGÊNCIAS PSIQUIÁTRICAS: REVISÃO INTEGRATIVA DOS RISCOS OCUPACIONAIS
}

\author{
Nursing in psychiatric emergencies: Integrating review of occupational risks \\ Enfermería en las urgencias psiquiátricas: Revisión integrative de los riesgos \\ ocupacionales
}

Juliana Araújo Cardoso ${ }^{1}$, Aline Costa de Oliveira ${ }^{2}$, Ivana Cavalcante Lemos de Sousa ${ }^{3}$, Roberta dos Santos Avelino ${ }^{4}$, Camilla de Oliveira Castelo Branco ${ }^{2}$, Jordana Fonseca Reis², Priscila de Macêdo Rocha $^{5}$, Aziz Moises Alves da Costa ${ }^{1}$, Teresa Amélia Carvalho de Oliveira ${ }^{1}$, Vitor Kauê de Melo Alves $^{1}$, Karllenh Ribeiro dos Santos ${ }^{1}$, Bruna Victoria da Silva Passos ${ }^{2}$, Ingrid Moura de Abreu², Daniel de Macêdo Rocha ${ }^{3^{*}}$.

\section{RESUMO}

Objetivo: Analisar nas evidências científicas os riscos ocupacionais decorrentes do cuidado de enfermagem nas urgências psiquiátricas. Métodos: Revisão integrativa da literatura realizada nas bases de dados Medical Literature Analysis and Retrieval System on-line, Cumulative Index to Nursing and Allied Health Literature, Web of Science ${ }^{\mathrm{TM}}$, SCOPUS, Literatura Latino-americana de Ciências da Saúde e Índice Bibliográfico Español en Ciencias de la Salud via Biblioteca Virtual em Saúde. A amostra foi composta por 12 estudos primários, publicados em inglês, português e italiano, sem delimitação temporal. Resultados: Predominaram estudos publicados em 2012, com delineamento observacional e nível de evidência 2C. Quanto aos riscos ocupacionais, estiveram relacionados tanto a violência física e quanto ao desenvolvimento de comorbidades psicopatológicas decorrentes da insegurança, de conflitos interpessoais, do medo ou do uso de substâncias psicoativas. Ainda, a síndrome de Burnout e a presença de condições estressantes constituíram fatores de risco para o comprometimento da qualidade da assistência. Conclusão: A assistência de enfermagem no setor de emergência psiquiátrica é rodeada de riscos ocupacionais que impactam negativamente no bem estar do profissional, assim como na prática de um cuidado com segurança, qualidade e efetividade.

Palavras-chave: Enfermagem Psiquiátrica, Riscos Ocupacionais, Serviços de Emergência Psiquiátrica.

\footnotetext{
ABSTRACT

Objective: To analyze in the scientific evidences the occupational risks arising from nursing care in psychiatric emergencies. Methods: Integrative literature review in the Medical Literature Analysis and Retrieval System online databases, Cumulative Index to Nursing and Allied Health Literature, Web of Science ${ }^{\mathrm{TM}}$, SCOPUS, Latin American Literature on Health Sciences and the Spanish Bibliographical Index on Health Sciences the Virtual Health Library. The sample consisted of 12 primary studies, published in English, Portuguese and Italian, without temporal delimitation. Results: Predominant studies published in 2012, with observational

1 Universidade Estadual do Piauí (UESPI), Teresina-Piauí. *E-mail: daniel_m.rocha@outlook.com.br.

2 Universidade Federal do Piauí (UFPI), Teresina-Piauí.

${ }^{3}$ Faculdade Santo Agostinho (FSA), Teresina-Piauí

${ }^{4}$ Fundação Municipal de Saúde (FMS), Teresina-Piauí.

${ }^{5}$ Faculdade de Tecnologia e Educação Superior (FATESP), Teresina-Piauí.
} 
design and level of evidence 2C. Regarding occupational risks, both physical violence and the development of psychopathological comorbidities related to insecurity, interpersonal conflicts, fear or the use of psychoactive substances were related. Still, Burnout syndrome and the presence of stressful conditions were risk factors for the quality of the care. Conclusion: Nursing care in the psychiatric emergency sector is surrounded by occupational risks that negatively impact the professional's well-being, as well as the practice of a care with safety, quality and effectiveness.

Key words: Psychiatric Nursing, Occupational Risks, Emergency Services Psychiatric.

\section{RESUMEN}

Objetivo: Analizar en las evidencias científicas los riesgos ocupacionales derivados del cuidado de enfermería en las urgencias psiquiátricas. Métodos: Revisión integrativa de la literatura en las bases de datos Medical Literature Analysis and Retrieval System on-line, Cumulative Index to Nursing and Allied Health Literature, Web of Science ${ }^{\mathrm{TM}}$, SCOPUS, Literatura Latinoamericana de Ciencias de la Salud e Índice Bibliográfico Español en Ciencias de la Salud de la salud vía Biblioteca virtual en sáude. La muestra consistió en 12 estudios primarios publicados en Inglés, portugués e italiano, sin delimitación temporal. Resultados: Predominaron estudios publicados en 2012, con delineamiento observacional y nivel de evidencia 2C. En cuanto a los riesgos ocupacionales, estuvieron relacionados tanto la violencia física y el desarrollo de comorbilidades psicopatológicas derivadas de la inseguridad, de conflictos interpersonales, del miedo o del uso de sustancias psicoactivas. Además, el síndrome de Burnout y la presencia de condiciones estresantes constituyeron factores de riesgo para el compromiso de la calidad de la asistencia. Conclusión: La asistencia de enfermería en el sector de emergencia psiquiátrica está rodeada de riesgos ocupacionales que impactan negativamente en el bienestar del profesional, así como en la práctica de un cuidado con seguridad, calidad y efectividad.

Palabras clave: Enfermería Psiquiátrica, Riesgos Laborales, Servicios de Urgencia Psiquiátrica.

\section{INTRODUÇÃO}

No Brasil, os serviços psiquiátricos passaram por diversas mudanças nos últimos anos, fato desencadeado pela Psiquiatria Reforma e alimentado por políticas de saúde mental que estabeleceram um novo modelo de assistência (ALVES SR, et al., 2018).

A Reforma Psiquiátrica, no final da década de 70 e início da década de 80, aconteceu em decorrência da crise do modelo hospitalocêntrico, regido por métodos pragmáticos, restringindo o doente mental do convívio com a família e a sociedade e impossibilitando-o de exercer sua cidadania. O hospital psiquiátrico, neste contexto arcaico, era a única alternativa de tratamento, o qual facilitava a cronicidade e exclusão dos doentes mentais. Os esforços movidos pela sociedade, familiares e profissionais da saúde, possibilitaram a mudança da forma de cuidar, de tratar e conviver com a problemática da loucura (OLIVEIRA LC, et al., 2018).

O modelo substitutivo ao manicômio propiciou o desenvolvimento de outras práticas, associadas ao estabelecimento de relacionamento terapêutico, em detrimento daquelas da psiquiatria tradicional, insuficientes para demandas de usuários, cujo resultado, ao longo dos anos, foi um alto grau de cronificação (MARTINS GCS, et al., 2018).

Para atuar em dispositivo de Atenção Psicossocial, o enfermeiro precisou dispor de outras estratégias de cuidar, como as tecnologias relacionais - vínculo, acolhimento, corresponsabilização. Atuar apenas com práticas tradicionais e tecnicistas, vinculadas ao modelo hospitalocêntrico, implicava risco de reproduzir o contexto manicomial em um serviço de reabilitação psicossocial. A utilização de ferramentas subjetivas faz parte de um cuidado complexo, porém menos tecnicista, em conformidade com o movimento da Reforma Psiquiátrica (MARTINS GCS, et al., 2018). 
Sabe-se que o enfermeiro e sua equipe representam, em termos numéricos, a maior força de trabalho das instituições hospitalares e a sua atuação, além das atividades assistenciais, direcionadas integralmente ao paciente e sua família, repercutem na dinâmica gerencial das instituições de saúde. Nessa perspectiva, a necessidade de dinamismo assistencial pode acarretar sobrecarga diária de trabalho e comprometer a qualidade de vida, as suas relações profissionais e o cuidado prestado, fazendo emergir conflitos que podem desvalorizar a sua atuação e diminuir o grau de satisfação, seja em âmbito individual ou multidisciplinar (BURIOLA AA, et al., 2016).

No ambiente hospitalar geral e psiquiátrico, a equipe de enfermagem é a que passa mais tempo em contato direto com os pacientes, porém nem todas as instituições oferecem condições laborais adequadas, o que acaba influenciando no grau de satisfação profissional relacionado ao trabalho (ALVES SR, et al., 2018).

Dessa forma, identificar o perfil dos enfermeiros que atuam em serviços de urgência e emergência psiquiátrica é relevante para a área de enfermagem psiquiátrica, pois, além de traçar um diagnóstico da situação, pode servir de subsídios para o planejamento de estratégias de formação e capacitação, visto que esses aspectos repercutem na assistência de enfermagem à pessoa em situação de urgência e emergência psiquiátrica, que, quando realizada com segurança, prontidão e qualidade, é capaz de determinar a aceitação e adesão do indivíduo atendido ao tratamento desse quadro (VARGAS D, et al., 2017).

Assim, para prestação de cuidados de qualidade na saúde mental é necessário que a enfermagem desenvolva competências não só específicas, mas, sobretudo, ampliadas daquelas definidas para o campo profissional da saúde (TAVARES C, et al., 2016).

Diante da magnitude do problema, este estudo tem como objetivo analisar nas evidências científicas os riscos ocupacionais decorrentes do cuidado de enfermagem nas urgências psiquiátricas.

\section{MÉTODOS}

Revisão integrativa da literatura fundamentada no referencial teórico de Whittemore e Knafl é conduzida por seis etapas de investigação, sendo elas: definição do tema e elaboração da questão de pesquisa; amostragem, busca na literatura e delimitação para a inclusão dos estudos; extração de dados; avaliação crítica dos resultados; síntese do conhecimento; e apresentação da revisão (WHITTEMORE R e KNAFL K, 2005).

Para elaboração da questão norteadora foi utilizado os elementos do acrônimo PICo, definindo-se os enfermeiros como população, os riscos ocupacionais como fenômeno de interesse e urgências e emergências psiquiátricas como contexto (LOCKWOOD C, et al., 2017). Assim, esta investigação foi conduzida a partir da seguinte pergunta: Quais as evidências científicas relacionadas aos riscos ocupacionais do enfermeiro nas urgências psiquiátricas?

O levantamento bibliográfico foi realizado entre os meses de outubro a dezembro de 2018 por meio da consulta as bases eletrônicas de dados Medical Literature Analysis and Retrieval System on-line (MEDLINE via PUBMED ${ }^{\circledR}$ ), Cumulative Index to Nursing and Allied Health Literature (CINAHL), Web of ScienceTM, SCOPUS, Literatura Latino-americana de Ciências da Saúde e Índice Bibliográfico Español en Ciencias de la Salud (LILACS e IBECS via Biblioteca Virtual em Saúde).

Os descritores controlados e não controlados, foram selecionados após consulta aos termos Descritores em Ciências da Saúde (DeCS), Medical Subject Headings (MESH) e List of Headings do CINAHL Information Systems, conforme descrito na figura 1. A combinação foi realizada por meio dos operadores booleanos OR e AND que possibilitou a construção da estratégia de busca, a qual foi adaptada de acordo com as especificidades de cada base. 
Figura 1 - Descritores controlados e não controlados utilizados para construção da estratégia de busca. Teresina, Piauí, Brasil, 2019.

\begin{tabular}{|c|c|c|}
\hline \multicolumn{3}{|r|}{ Descritores em Ciências da Saúde - DeCS } \\
\hline \multicolumn{2}{|r|}{ Descritor controlado } & Descritor não controlado \\
\hline $\mathbf{P}$ & Enfermagem Psiquiátrica & Enfermagem Psiquiátrica. \\
\hline $\mathbf{I}$ & $\begin{array}{l}\text { Riscos Ocupacionais } \\
\text { Fatores de Risco }\end{array}$ & $\begin{array}{l}\text { Riscos Ocupacionais; Condições Inseguras no Trabalho; Risco } \\
\text { Ocupacional; Fatores de Risco; Fator de Risco. }\end{array}$ \\
\hline Co & $\begin{array}{l}\text { Serviços de Emergência } \\
\text { Psiquiátrica } \\
\text { Emergências } \\
\text { Socorro de Urgência }\end{array}$ & $\begin{array}{l}\text { Serviços de Emergência Psiquiátrica; Serviços de Urgência } \\
\text { Psiquiátrica; Emergências; Urgência; Urgências; Emergência; } \\
\text { Atendimento de Emergência; Atendimento de Urgência; Centros de } \\
\text { Atendimento de Urgência; Clínicas de Atendimento de Urgência; } \\
\text { Socorro de Urgência. }\end{array}$ \\
\hline \multicolumn{3}{|r|}{ LILACS e IBECS via BVS } \\
\hline \multicolumn{3}{|c|}{$\begin{array}{l}\text { (tw:((mh:("Enfermagem Psiquiátrica")) OR (tw:("Enfermagem Psiquiátrica")))) AND (tw:((mh:("Riscos } \\
\text { Ocupacionais")) OR (tw:("Riscos Ocup4acionais" )) OR (mh:("Fatores de Risco")) OR (tw:("Fatores de } \\
\text { Risco")) OR (tw:("Condições Inseguras no Trabalho")) OR (tw:("Risco Ocupacional")) OR (tw:("Fator de } \\
\text { Risco")) )) AND (tw:((mh:("Serviços de Emergência Psiquiátrica")) OR (tw:("Serviços de Emergência } \\
\text { Psiquiátrica")) OR (mh:(Emergências)) OR (tw:(Emergências)) OR (mh:("Socorro de Urgência")) OR } \\
\text { (tw:("Socorro de Urgência")) OR (tw:("Serviços de Urgência Psiquiátrica")) OR (tw:(Urgência)) OR } \\
\text { (tw:(Urgências)) OR (tw:(Emergência)) OR (tw:("Atendimento de Emergência")) OR (tw:("Atendimento de } \\
\text { Urgência")) OR (tw:("Centros de Atendimento de Urgência")) OR (tw:("Clínicas de Atendimento de } \\
\text { Urgência")))) }\end{array}$} \\
\hline \multicolumn{3}{|r|}{ MESH e list CINAHI } \\
\hline $\mathbf{P}$ & Psychiatric Nursing & $\begin{array}{l}\text { Psychiatric Nursing; Mental Health Nursing; } \\
\text { Nursing, Mental Health; Nursing, Psychiatric; Psychosocial Nursing. }\end{array}$ \\
\hline $\mathbf{I}$ & Risk Factors & Risk Factors; Occupational Risks; Risk Factor. \\
\hline Co & $\begin{array}{l}\text { Emergency } \\
\text { Services Psychiatric } \\
\text { Emergencies }\end{array}$ & $\begin{array}{l}\text { Emergency Services, Psychiatric; Psychiatric Emergency Services; } \\
\text { Psychiatric Emergency Service; Emergency Psychiatric Services; } \\
\text { Emergencies; Emergency; Emergency Relief. }\end{array}$ \\
\hline \multicolumn{3}{|r|}{ P AN I AND Co } \\
\hline \multicolumn{3}{|r|}{ MEDLINE via PUBMED } \\
\hline \multicolumn{3}{|c|}{$\begin{array}{l}\text { ((((((("Psychiatric Nursing"[Mesh]) OR "psychiatric nursing") OR "mental health nursing") OR "nursing } \\
\text { mental health") OR "nursing, psychiatric") OR "psychosocial nursing")) AND (((("Risk Factors"[Mesh]) OR } \\
\text { "risk factors") OR "occupational risks") OR "risk factor")) AND ((((((((("Emergency Services, } \\
\text { Psychiatric"[Mesh]) OR "Emergencies"[Mesh])) OR "emergency services, psychiatric") OR "emergencies") } \\
\text { OR "psychiatric emergency services") OR "psychiatric emergency service") OR "emergency psychiatric } \\
\text { services") OR "emergency") OR "emergency relief") }\end{array}$} \\
\hline \multicolumn{3}{|r|}{ SCOPUS } \\
\hline \multicolumn{3}{|c|}{$\begin{array}{l}\text { ( ( TITLE-ABS-KEY ( "Psychiatric Nursing" ) OR TITLE-ABS-KEY ( "Mental Health Nursing" ) OR TITLE- } \\
\text { ABS-KEY ( "Nursing, Mental Health" ) OR TITLE-ABS-KEY ( 4"Nursing, Psychiatric" ) OR TITLE-ABS-KEY } \\
\text { ( "Psychosocial Nursing" ) ) ) AND ( ( TITLE-ABS-KEY ( "Risk Factors" ) OR TITLE-ABS-KEY } \\
\text { "Occupational Risks") OR TITLE-ABS-KEY ( "Risk Factor" ) )) AND ( ( TITLE-ABS-KEY ( "Emergency } \\
\text { Services, Psychiatric") OR TITLE-ABS-KEY ( "Psychiatric Emergency Services" ) OR TITLE-ABS-KEY } \\
\text { "Psychiatric Emergency Service" ) OR TITLE-ABS-KEY ( "Emergency Psychiatric Services" ) OR TITLE- } \\
\text { ABS-KEY ( "Emergencies" ) OR TITLE-ABS-KEY ( "Emergency" ) OR TITLE-ABS-KEY ( "Emergency } \\
\text { Relief" ) ) ) }\end{array}$} \\
\hline
\end{tabular}




\section{WEB OF SCIENCE}

(TS=("Psychiatric Nursing") OR TS=("Mental Health Nursing") OR TS=("Nursing, Mental Health") OR TS=("Nursing, Psychiatric") OR TS=("Psychosocial Nursing")) AND (TS=("Risk Factors") OR TS=("Occupational Risks") OR TS=("Risk Factor")) AND (TS=("Emergency Services, Psychiatric") OR TS=("Psychiatric Emergency Services") OR TS=("Psychiatric Emergency Service") OR TS=("Emergency Psychiatric Services") OR TS=("Emergencies") OR TS=("Emergency") OR TS=("Emergency Relief"))

\section{CINAHL}

( (MH "Psychiatric Nursing") OR "Psychiatric Nursing" OR "Mental Health Nursing" OR "Nursing, Mental Health" OR "Nursing, Psychiatric" OR "Psychosocial Nursing" ) AND ( (MH "Risk Factors") OR "Risk Factors" OR "Risk Factor" OR "Occupational Risks" ) AND ( (MH "Emergency Services, Psychiatric") OR "Emergency Services, Psychiatric" OR "Psychiatric Emergency Services" OR "Psychiatric Emergency Service" OR "Emergency Psychiatric Services" OR (MH "Emergencies") OR "Emergencies" OR (MH "Psychiatric Emergencies") OR "Psychiatric Emergencies" OR "Emergency" OR "Emergency Relief" )

Fonte: Cardoso JA, et al, 2019.

Para inclusão foram definidos os seguintes critérios: estudos primários, sem delimitação temporal ou de idioma e que descrevessem os desafios assistenciais enfrentados pelo enfermeiro no cuidado às urgências psiquiátricas. Os critérios de exclusão focaram-se nos artigos duplicados.

Os artigos foram acessados por meio do portal de periódicos da Coordenação de Aperfeiçoamento de Pessoal de Nível Superior e a busca, seleção e inclusão realizada por dois revisores de forma independente que procederam com a leitura de títulos e resumos de modo a garantir maior rigor metodológico e fidedignidade dos resultados.

Foram recuperados 64 produções, dentre as quais 20 atenderam aos critérios de inclusão, sendo selecionadas para o estudo. Destaca-se que, 8 artigos foram excluídos por duplicidade nas bases de dados, resultando na amostra de 12 publicações. A Figura 2 descreve o percurso realizado para identificação, inclusão e exclusão dos estudos, segundo base consultada.

Figura 2 - Percurso para identificação, inclusão e exclusão nas bases eletrônicas investigadas.

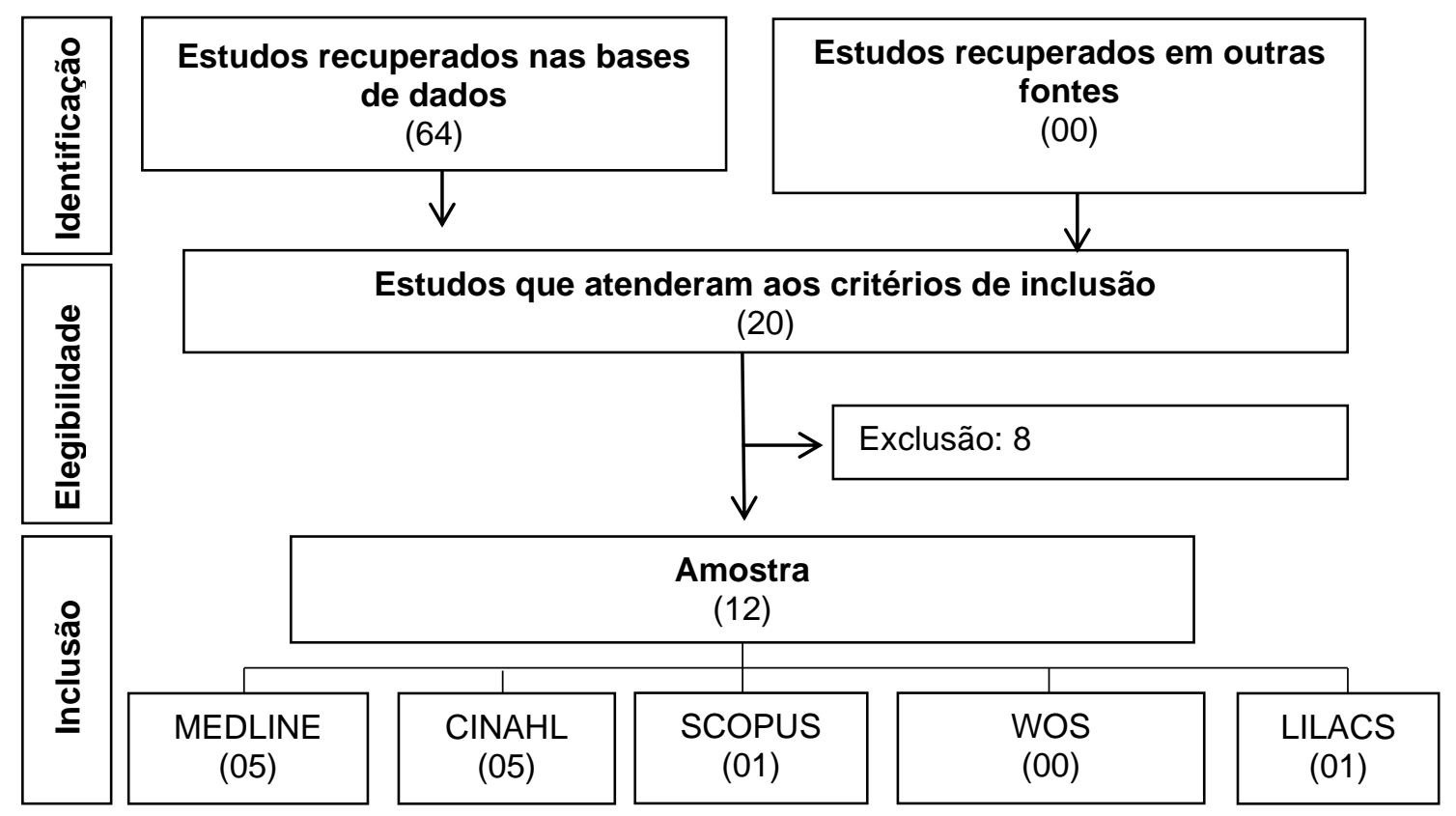

Fonte: Cardoso JA, et al, 2019. 
A coleta dos dados foi realizada com auxílio de um instrumento próprio que contemplou variáveis relacionadas a identificação dos estudos (autores, país e ano de publicação), aspectos metodológicos (delineamento), principais resultados, desfechos, conclusões e nível de evidência.

Para análise do Nível de Evidência (NE) foram seguidas as recomendações propostas pelo Oxford Centre for Evidence-based Medicine que classifica as evidências de acordo com o delineamento metodológico: 1A revisão sistemática de ensaios clínicos controlados randomizados; $1 \mathrm{~B}$ - ensaio clínico controlado randomizado com intervalo de confiança estreito; $1 \mathrm{C}$ - resultados terapêuticos do tipo "tudo ou nada"; $2 \mathrm{~A}$ - revisão sistemática de estudos de coorte; $2 \mathrm{~B}$ - estudo de coorte (incluindo ensaio clínico randomizado de menor qualidade); $2 \mathrm{C}$ - observação de resultados terapêuticos ou estudos ecológicos; $3 \mathrm{~A}$ - revisão sistemática de estudos caso-controle; 3B - estudo caso-controle; 4 - relato de casos (incluindo coorte ou caso-controle de menor qualidade); 5 - opinião de especialistas (OXFORD, 2009).

A análise crítica e a síntese dos resultados foi realizada de forma descritiva, o que possibilitou a classificação dos estudos por similaridade semântica.

\section{RESULTADOS}

Dentre os artigos incluídos, verificou-se que o ano de publicação variou de 1997 a 2017, sendo 2012 o de maior produção com 3 artigos. Quanto ao idioma, 10 foram publicados em inglês, 1 em português e 1 em italiano. Em relação às bases de dados, 5 foram identificados na MEDLINE/PubMed, 1 na LILACS, 5 na CINAHL e 1 na SCOPUS.

No que se refere ao delineamento metodológico, seis foram transversais, três descritivos, uma revisão sistemática e duas revisões bibliográficas, em que a maioria apresentou nível de evidência $2 \mathrm{C}$. A síntese do conhecimento está apresentada na Figura 3, conforme autor principal, país, ano de publicação, delineamento, nível de evidência e principais resultados, desfechos e conclusões.

Figura 3 - Síntese dos estudos incluídos. Teresina, PI, Brasil, 2019.

\begin{tabular}{|c|c|l|}
\hline Autor, país e ano & Delineamento - NE & \multicolumn{1}{|c|}{ Principais resultados, desfechos e conclusões } \\
\hline $\begin{array}{c}\text { BLANDO, JD. EUA, } \\
2012\end{array}$ & Transversal - 2C & Insegurança e abuso verbal. \\
\hline $\begin{array}{c}\text { DARBRO, N. EUA, } \\
2012\end{array}$ & Transversal - 2C & $\begin{array}{l}\text { Transtornos mental e comportamental relacionados ao uso de } \\
\text { substâncias psicoativas, estresse e conflitos interpessoais. }\end{array}$ \\
\hline $\begin{array}{c}\text { CAHUL, CD. EUA, } \\
2009\end{array}$ & Descritivo - 2C & Comportamento violento dos pacientes. \\
\hline $\begin{array}{c}\text { FERRI, P. Itália, } \\
2011\end{array}$ & Transversal - 2C & Violência física e verbal. \\
\hline $\begin{array}{c}\text { WHITTINGTON, R. } \\
\text { Inglaterra, 1997 }\end{array}$ & Descritivo - 2C & $\begin{array}{l}\text { Risco de violência relacionada ao trabalho, ameaças e } \\
\text { agressões. }\end{array}$ \\
\hline $\begin{array}{c}\text { NUNES, MBG. } \\
\text { Brasil. 2012 }\end{array}$ & Descritivo - 2C & Estresse, cansaço e desvaloriação. \\
\hline $\begin{array}{c}\text { PICH, JV. Austrália. } \\
2017\end{array}$ & Transversal - 2C & Violência e abuso verbal. \\
\hline $\begin{array}{c}\text { MARTINEZ, AJS. } \\
\text { EUA, 2016 }\end{array}$ & $\begin{array}{c}\text { Revisão } \\
\text { bibliográfica - 2C }\end{array}$ & $\begin{array}{l}\text { Insegurança, agressão verbal, comportamentos abusivos, } \\
\text { medo de retaliação, violência física e psicológica. }\end{array}$ \\
\hline $\begin{array}{c}\text { CHILD, RJH. EUA, } \\
2010\end{array}$ & Revisão & $\begin{array}{l}\text { Violência, medo, baixa moral da equipe e transtornos de } \\
\text { ansiedade. }\end{array}$ \\
\hline $\begin{array}{c}\text { IMAl, H. Japão } \\
2004\end{array}$ & Transversal - 2C & $\begin{array}{l}\text { Síndrome de burnout associada a carga excessiva de } \\
\text { trabalho. }\end{array}$ \\
\hline $\begin{array}{c}\text { SEVRINSSON, E. } \\
\text { Noruega, 2001 }\end{array}$ & Transversal - 2C & $\begin{array}{l}\text { Síndrome de burnout associada a insatisfação, estresse e } \\
\text { experiências com deficiência além do sofrimento moral. }\end{array}$ \\
\hline $\begin{array}{c}\text { EDWARD, K. } \\
\text { Austrália, 2014 }\end{array}$ & $\begin{array}{c}\text { Revisão } \\
\text { sistemática -2A }\end{array}$ & $\begin{array}{l}\text { Impacto psicológico pós incidente como agressão verbal e/ou } \\
\text { física. }\end{array}$ \\
\hline
\end{tabular}

Fonte: Cardoso JA, et al, 2019. 


\section{DISCUSSÃO}

\section{Fatores de Risco}

A assistência de enfermagem psiquiátrica é marcada por riscos ocupacionais, dentre eles o mais identificado nos estudos foi a violência que pode estar associada a idade, ao sexo e a história de um relacionamento abusivo atual, ao passado do paciente ou a experiência de enfermagem. Enfermeiros que são mais jovens e com menos experiência foram encontrados com altas taxas de violência no local de trabalho, podendo estar relacionado ao fato de que profissionais mais experientes podem ocupar cargos gerenciais e ter menor contato com o paciente (CHILD RJH e MENTES JC, 2010). Enfermeiros com idade inferior a 45 anos e estudantes de enfermagem, apresentam dificuldades ou limitações para identificar sinais de alerta para situações de risco ou maior vulnerabilidade (PICH JV, et al., 2017).

Os estudos relatam que as mulheres são mais propensas a serem agredidas no local de trabalho, porém a predominância do sexo feminino na equipe de enfermagem torna influencia diretamente para este resultado (CHILD RJH e MENTES JC, 2010).

Outro fator de risco está associado ao fácil acesso ao setor de urgência psiquiátrica, bem como ao perfil de atendimento caracterizado pelo aumentado do ruído acarretando estresse, diminuição da segurança e longos tempos de espera (CHILD RJH e MENTES JC, 2010).

Verificou-se também que enfermeiros do turno da noite e fim de semana estão mais propensos a sofrer agressão no local de trabalho. Em parte, isso pode ser atribuído ao relativo isolamento em que esses enfermeiros estão trabalhando e o número de pessoal mais baixos durante esse período (EDWARD K, et al., 2014).

Os achados dessa revisão constataram que a exposição à violência pode ter um impacto na maneira como os enfermeiros interagem com seus pacientes, e isso inclui sentir menos empatia e um declínio na qualidade do atendimento ofertado (PICH JV, et al., 2017).

\section{Tipos de Violência}

Quanto aos tipos de violência, pode-se observar que os enfermeiros psiquiátricos tem sofrido frequentemente agressões físicas ou psicológicas e verbais (FERRI P, et al., 2011). Os tipos mais comuns de abuso verbal contra enfermeiros incluiu gritar, ser amaldiçoado, ser intimidado e ser assediado com linguagem sexual e insinuações. No caso de agressão como intimidação e abuso verbal, isso incluiu ataques sobre questões de competência e assistencial (EDWARD K, et al., 2014).

As agressões físicas podem ter manifestações externas, mas a literatura demonstra cada vez mais que essa situação tem ramificações ainda mais negativas e duradouras, variando de acordo com o diagnóstico e condições psiquiátricas apresentadas durante o atendimento inicial (CHILD RJH e MENTES JC, 2010).

Os estudos relatam como impactos da experiência de agressão a longo prazo, a perda de confiança, a ausência no trabalho, a perda de boa relação de trabalho com os colegas, a automedicação (incluindo drogas e álcool) e até mesmo o abandono das atividades laborais (EDWARD K, et al., 2014).

Salienta-se que o impacto da violência apresenta longo alcance e afeta os enfermeiros psicologicamente, fisicamente e profissionalmente se constituindo como condição geradora de morbidade. $O$ abuso verbal pode causar estresse e trauma psicológico significativo, mesmo se não houver ferimento físico, e isso pode persistir por até 12 meses após o incidente (PICH JV, et al., 2017).

\section{Síndrome de Burnout e Fatores de Estresse}

Ainda, estudos identificaram que a prevalência de Burnout é significativamente maior para enfermeiros psiquiátricos do que os enfermeiros envolvidos em outros serviços de saúde pública. O aumento da frequência 
de serviços que exigem horas extras de urgência no trabalho elevou a prevalência de Burnout nessa categoria de enfermeiros (IMAI H, et al., 2004).

Os artigos reforçam que os serviços de saúde mental diferem de outros serviços de saúde pública no que diz respeita aos termos da natureza, dos casos que requerem horas extras, e que os serviços de saúde mental envolvem casos difíceis que são mais propensos a causar Burnout (IMAI H, et al., 2004).

Pôde-se observar que enfermeiros que são submetidos a alta carga de trabalho, baixa influência sobre suas atribuições, oportunidades limitadas de melhoria de habilidades e pouco apoio de seus supervisores experimentam um estresse moral. Com isso, fica evidente uma tensão considerável que resulta na deterioração das condições de trabalho e diminuição da satisfação no trabalho (SEVRINSSON E e HUMMELVOLL JK, 2001).

O sofrimento moral dos enfermeiros é um fator que foi previamente relacionado ao Burnout, e faz com que seja necessário explorar as consequências do comportamento que é associado com Burnout, bem como o relacionamento entre tal comportamento e satisfação do paciente com o cuidado prestado pelos enfermeiros (SEVRINSSON E e HUMMELVOLL JK, 2001).

Diante disso, foi possível observar a necessidade de melhoria no ambiente de trabalho no setor de urgências psiquiátricas a fim de evitar o Burnout entre os enfermeiros (IMAI H, et al., 2004). Outro estudo mostra que problemas no trabalho, no ambiente e interpessoal foram preditores para o estresse revelados pelos profissionais de enfermagem. Entretanto, a maioria não associa estes fatores às alterações psicofisiológicas que relatam possuir e como causa de falta ao trabalho por doença (NUNES MBG, 2000).

\section{Uso de Substâncias Psicoativas}

O uso de substâncias psicoativas foi uma prática frequente entre os enfermeiros psiquiátricos, estando relacionada ao fácil acesso e pela pronta disponibilidade de medicamentos, associadas a deficiências no gerenciamento e dispensação dessas substâncias. A falta de educação sobre o processo de dependência e seus sinais e sintomas é um dos mais profundos e negligenciados fatores de risco para enfermeiros (DARBRO $\mathrm{N}$ e MALLIARAKIS KD, 2012).

Além desses fatores algumas atitudes dos enfermeiros também foram destacadas, como a visão dos enfermeiros sobre a substância como um meio aceitável de lidar com os problemas da vida e uma maneira de promover o prazer, o conforto e a capacidade de lidar, se dar bem e conviver. Devido sua formação acadêmica e observações, também foi destacada a crença no uso das drogas como um meio de promover cura (DARBRO N e MALLIARAKIS KD, 2012).

\section{Subnotificações dos Casos de Violência}

Embora a subnotificação da violência no local de trabalho limita a plena compreensão da extensão deste problema, em contextos de cuidados de saúde, os enfermeiros possuem a maior taxa de ataques violentos de todos os profissionais de saúde (CHILD RJH e MENTES JC, 2010).

Os achados dessa revisão destacaram que os enfermeiros não relatam a violência porque sentem que serão visto como incompetente, como tendo provocado o incidente, ou como causadores de problemas. Outras possíveis teorias por que os enfermeiros não relatam a violência sofrida incluem o pouco conhecimento sobre os procedimentos de relatórios existentes, o entorpecimento do enfermeiro vítima devido a exposições da sociedade (MARTINEZ AJS, 2016; CHILD RJH e MENTES JC, 2010; IMAI H, et al., 2004).

Diante dos estudos analisados, pôde-se observar um alto nível de não notificação por parte dos enfermeiros após incidentes agressivos (verbais e físicos). O não relato continua a ser uma preocupação organizacional e possíveis barreiras foram identificadas como contribuindo para a falta de relatos dos profissionais que vivenciaram agressão. Essas barreiras foram identificadas como: Falta de (ou falta de 
clareza) política / procedimento de comunicação de incidentes; Apoio administrativo deficiente ou ausente para o indivíduo pós incidente; Enfermeiros que tiveram uma experiência anterior de não ação pós incidente agressivo e optando por ignorar o incidente; Medo de consequências ocupacionais adversas, tais como percebido como não lidando e não sendo capaz de se envolver bem com os pacientes (EDWARD K, et al., 2014).

Observou-se também como barreiras que contribuíram para o fenômeno de subnotificação de incidentes de violência no local de trabalho o medo de retaliação dos enfermeiros por parte de seus empregadores e pouco apoio da administração (MARTINEZ AJS, 2016). Inclui-se ainda o medo dos enfermeiros de perder seus empregos e a crença de que relatar esses incidentes não mudaria nenhuma prática atual contra a violência sofrida no local de trabalho (MARTINEZ AJS, 2016).

\section{CONCLUSÃO}

Foi possível evidenciar que os a emergência psiquiátrica apresenta riscos ocupacionais, na maioria das vezes relacionadas a situações de violência sejam elas físicas ou psicológicas. Nesse sentido, verificou-se que os profissionais vítimas de agressão tendem a tornar-se mais apáticos em relação aos pacientes e menos cuidadoso, podendo refletir no mau atendimento e menor satisfação com o ambiente de trabalho. Assim, observa-se a necessidade de melhoria no ambiente laboral a fim de evitar danos maiores à saúde dos profissionais que prestam essa assistência, em especial ao enfermeiro, que tem contato mais próximo com o paciente.

\section{REFERÊNCIAS}

1. ALVES SR, et al. Serviços de saúde mental: percepção da enfermagem em relação à sobrecarga e condições de trabalho. J. Res. Fundam. Care, 2018; 10(1): 25-29.

2. ALVES SR, SANTOS RP, YAMAGUCHI UM. Enfermagem em Serviços de Saúde Mental: Percepção sobre Satisfação Profissional e Condições de Trabalho. Revista de Enfermagem do Centro-Oeste Mineiro. 2018; 8(e1852): 1-7.

3. BLANDO JD, et al. Impact of hospital security programmes and workplace aggression on nurse perceptions of safety. Journal of Nursing Management, 2013; 21: 491-498.

4. BURIOLA AA, et al. Atuação do enfermeiro no serviço de emergência psiquiátrica: Avaliação pelo método de quarta geração. Texto Contexto Enferm. Florianópolis (SC), 2016; 25(1): 1-9.

5. CAHUL CD, et al. Inpatient Management of Violent Behavior: Nursing Prevention and Intervention, Issues in Mental Health Nursing. 1991; 12(3): 239-252.

6. OXFORD. Centre for Evidence-Based Medicine. Levels of evidence [Internet]. 2009. Available from: http://www.cebm.net/oxford-centre-evidence-based-medicine-levels-evidence-march-2009/

7. CHILD RJH, MENTES JC. Violence Against Women: The Phenomenon of Workplace Violence Against Nurses. Issues in Mental Health Nursing. 2010; 31: 89-95.

8. DARBRO N, MALLIARAKIS KD. Substance Abuse: Risks Factors and Protective Factors. Journal of Nursing Regulation. 2012; 3(1): 44-48.

9. EDWARD K, et al. Nursing and aggression in the workplace: a systematic review. British Journal of Nursing. 2014; 23(12): 653-4.

10. FERRI P, et al. Aggressive behavior toward nursing staff in three different health care settings. Professioni Infermieristiche. 2011; 64(3): 143-150.

11. IMAI H, et al. Burnout and work environments of public health nurses involved in mental health care. Occup Environ Med. 2004; 61: 764-768. 
12. LOCKWOOD C, et al. Chapter 2: Systematic reviews of qualitative evidence. In: Aromataris E, Munn Z (Editors). Joanna Briggs Institute Reviewer's Manual. The Joanna Briggs Institute, 2017. Available from https://reviewersmanual.joannabriggs.org/

13. MARTINEZ AJS. Managing workplace violence with evidence - Based interventions. A literature review. Journal of Psychosocial Nursing. 2016; 54(9): 31-36.

14. MARTINS GCS, et al. Estratégias de cuidado adotadas por enfermeiros na implantação de Centros de Atenção Psicossocial. Rev Rene. 2018; 19(e33319): 1-8.

15. NUNES MBG. Stress in nursing workers: study in a psychiatry unit. 110 f (Dissertação de mestrado). Universidade do Estado do Rio de Janeiro. Rio de Janeiro, 2000.

16. OLIVEIRA LC, et al. Dificuldades nos atendimentos às urgências psiquiátricas no serviço de atendimento móvel de urgência. Enferm. Foco. 2018; 9(4): 18-22.

17. $\mathrm{PICH}$ JV, et al. Antecedents and precipitants of patient-related violence in the emergency department: Results from the Australian VENT Study (Violence in Emergency Nursing and Triage). Australasian Emergency Nursing Journal. 2017; 20(3):107-113.

18. SEVRINSSON E, HUMMELVOLL JK. Factors influencing job satisfaction and ethical dilemas in acute psychiatric care. Nursing and Health Sciences. 2001; 3: 81-90.

19. TAVARES $\mathrm{C}$, et al. Competências específicas do enfermeiro de saúde mental enfatizadas no ensino de graduação em enfermagem. Revista Portuguesa de Enfermagem de Saúde Mental. 2016; 4: 25-32.

20. VARGAS D, et al. Enfermeiros de serviços de urgência e emergência psiquiátrica: Análise de perfil profissional e educacional. Cogitare Enferm. 2017; 22(4): 1-9.

21. WHITTEMORE R, KNAFL K. The integrative review: updated methodology. J Adv Nurs. 2005; 52(5): 546-553.

22. WHITTINGTON R. Violence to nurses: prevalence and risk factors. Nursing Standard. 1997; 12(5): 49-56. 